\title{
Tratamento cirúrgico no acidente vascular cerebral hemorrágico: afinal, o que há de evidências?
}

\author{
Robson Luis Oliveira de Amorim¹, Wellingson Silva Paiva', Eberval Gadelha Figueiredo', \\ Marcelo Prudente Espírito Santo ${ }^{1}$, Almir Ferreira de Andrade ${ }^{2}$, Manoel Jacobsen Teixeira ${ }^{3}$
}

Divisão de Neurocirurgia do Hospital das Clínicas da Faculdade de Medicina da Universidade de São Paulo (HC-FMUSP), São Paulo, SP.

\begin{abstract}
RESUMO
Mais da metade dos pacientes com hematoma cerebral espontâneo evolui desfavoravelmente. O controle inadequado da pressão arterial sistêmica é uma das principais causas para esse quadro dramático. Várias pesquisas clínicas visando ao controle da expansão do hematoma têm se mostrado satisfatórias, entretanto pouco houve de avanço no tratamento cirúrgico dessas condições. Há inúmeras variáveis que devem ser avaliadas em um paciente com hematoma intraparenquimatoso espontâneo, e esta revisão visa sistematizar o tratamento cirúrgico baseado em evidências.

\section{PALAVRAS-CHAVE}

Acidente vascular cerebral. Hemorragia intracerebral.

ABSTRACT

Surgical treatment of spontaneous cerebral hematoma: are there any evidence? More than half of patients with spontaneous cerebral hematoma have a poor outcome. The inadequate control of the systemic blood pressure is one of the main causes for this dramatic picture. Several clinical researches seeking the control of the hematoma expansion have shown satisfactory findings, however, little progress was demonstrated in the surgical treatment of those conditions. There are countless variables that should be appraised in a patient with spontaneous cerebral hematoma, and this review intends to systematize the surgical treatment based on evidences.
\end{abstract}

\section{KEY WORDS}

Stroke. Intracerebral hemorrhage.

\section{Introdução}

A hemorragia intracerebral espontânea responde por até $10 \%$ de todos os casos de acidentes vasculares encefálicos (AVE) e em cerca de $50 \%$ a evolução é fatal. Há ainda muita controvérsia a respeito do tratamento dessa condição e nem o tratamento clínico ou o cirúrgico mostraram-se eficazes o suficiente para diminuir a morbimortalidade. Os principais fatores relacionados com evolução desfavorável descritos na literatura são volume do hematoma, escore de admissão na Escala de Glasgow, extensão hemorrágica intraventricular ou subaracnoidea, hidrocefalia, uso de agentes anticogulantes e magnitude do edema associado. ${ }^{5,6,8,9,12,14}$ Teoricamente, a redução do tamanho do hematoma diminui o efeito de massa, reduzindo a pressão intracraniana (PIC) e evitando a progressão do edema e morte celular. . $^{1,38,39,41}$

\section{0 tratamento cirúrgico $\mathrm{e}$ cuidados pré-operatórios}

O tratamento cirúrgico clássico é a craniotomia com drenagem do hematoma. Para manejo da PIC, a

1. Divisão de Neurocirurgia do Hospital das Clínicas da Faculdade de Medicina da Universidade de São Paulo (HC-FMUSP), São Paulo, SP.

2. Coordenador do Pronto-Socorro Neurocirúrgico do HC-FMUSP.

3. Professor titular da disciplina de Neurocirurgia da FMUSP. 
ventriculostomia é uma alternativa importante em casos selecionados. Infelizmente, até o momento não se sabe exatamente o limite para definição de que o tratamento cirúrgico pode mudar o curso da doença. $\mathrm{O}$ tratamento inicial visa manter a permeabilidade das vias aéreas e assegurar oxigenação adequada. A investigação de distúrbios plaquetários ou coagulopatias torna-se imperativa, visto a possibilidade clínica de conter a expansão do hematoma, por meio da administração de plaquetas, plasma fresco congelado, vitamina $\mathrm{K}$ e/ou complexo protrombínico. Esses pacientes são idealmente conduzidos em ambiente de UTI, com o objetivo de manter os níveis pressóricos estabilizados e o nível de consciência monitorado. Alguns estudos revelam que a hipertensão arterial descontrolada após o evento hemorrágico é um fator de mau prognóstico. ${ }^{13,23,31,32,34}$

\section{0 paciente comatoso}

Nos pacientes comatosos, a monitorização da PIC deve ser realizada. Nos pacientes sem coagulopatias, com desvio das estruturas da linha mediana, ainda sem indicação para tratamento cirúrgico por meio de craniotomia, o monitor intraparenquimatoso é uma opção. A monitorização da PIC mediante transdutor intraventricular permite tanto o diagnóstico da hipertensão intracraniana (HIC) quanto o tratamento dessa condição. Os pacientes com postura específica ou que apresentam escore 3 na Escala de Coma de Glasgow (ECGlasgow) à admissão geralmente têm prognóstico muito reservado, e a conduta cirúrgica deve ser tomada apenas em casos selecionados no caso de lesões supratentoriais.

\section{Em quem intervir cirurgicamente?}

Os casos clássicos, em que a terapia cirúrgica se mostra eficaz, são os pacientes com menos de 60 anos, com hemorragias superficiais em hemisfério direito, que pioram neurologicamente. Pacientes com escore menor ou igual a 14 na ECGlasgow e com hematoma supratentorial maior que $5 \mathrm{~cm}$ de diâmetro ou $30 \mathrm{~cm}^{3}$ podem beneficiar-se da cirurgia, em contraste com aqueles com escore inferior a 14 e com hematomas menores. ${ }^{23}$ Entretanto, a cirurgia inequívoca e as diretrizes para intervenção não são bem claras, pois a maioria dos estudos randomizados tem sido inconclusiva. Apesar de o tamanho do hematoma influenciar na decisão cirúrgica, a acessibilidade ao coágulo é mais importante.
Os hematomas lobares ou aqueles localizados próximos à superfície influenciam mais na decisão cirúrgica do que aqueles localizados no putâmen ou no tálamo. ${ }^{7}$ A extensão intraventricular do hematoma está associada a uma mortalidade de $45 \%$, comparada com $9 \%$ daqueles hematomas restritos ao parênquima ${ }^{22,35}$ (Quadro 1), e a hidrocefalia é um preditor independente de morte após AVE hemorrágico (AVEH). ${ }^{11}$

Quadro 1

Fatores de mau prognóstico em AVE hemorrágico

\footnotetext{
- Idade maior que 60 anos

- Torpor ou coma na admissão

• Extensão intraventricular do hematoma

- Volume do edema perilesional

- Hidrocefalia

- Uso de antiplaquetários ou anticoagulantes
}

\section{Quando indicar a cirurgia?}

Alguns autores ${ }^{24,28}$ têm sugerido melhora da função neurológica em pacientes operados entre 12 e 24 horas de evolução. Kaneko e cols. ${ }^{21}$ evidenciaram melhor prognóstico (mortalidade de $7 \%$ em seis meses) em pacientes operados até sete horas desde o evento ictal. Entretanto, Morgenstern e cols. ${ }^{27}$ demonstraram aumento na taxa de ressangramento em pacientes operados com menos de quatro horas do ictus. $\mathrm{O}$ único estudo randomizado ${ }^{42}$ direcionado a responder essa questão mostrou que pacientes operados precocemente têm tendência a melhor prognóstico (não estatisticamente significante), e o estudo multicêntrico internacional STICH não revelou nenhum efeito benéfico em termos de prognóstico com a cirurgia precoce após hemorragias supratentoriais. ${ }^{25}$

\section{Craniotomia}

A craniotomia é o procedimento cirúrgico mais utilizado nos casos de AVEH. Juvela e cols., ${ }^{19}$ em um estudo em que foi comparada a cirurgia com o tratamento clínico em pacientes com déficits neurológicos severos ou rebaixamento do nível de consciência, não encontraram diferença estatística significativa. No grupo cirúrgico, a mortalidade foi de $46 \%$, e $50 \%$ tornaram-se dependentes, enquanto no grupo clínico $38 \%$ morreram e $42 \%$ ficaram com sequelas graves. Em um estudo controlado, ${ }^{36}$ também não foi encontrada diferença com relação ao prognóstico a longo prazo. Em um trial randomizado (STICH) em que 
foram avaliados 1.033 pacientes, dentre os pacientes submetidos ao tratamento cirúrgico, $26 \%$ tiveram prognóstico favorável comparados com $24 \%$ dos pacientes randomizados para o tratamento conservador. ${ }^{25}$ Até o momento, nenhum estudo controlado demonstrou a superioridade do tratamento cirúrgico em relação aos cuidados clínicos intensivos.

\section{Hemorragia putaminal}

A localização mais comum das hemorragias intracranianas de origem hipertensiva é a de tratamento mais controverso, por causa da profundidade da lesão. A extensão lateral desse hematoma comprime a ínsula, enquanto sua extensão medial pode causar inundação ventricular. Apesar de não randomizado, Kanaya e Kuroda $^{20}$ estudaram 7.010 pacientes e encontraram que, nos pacientes torporosos ou comatosos e com hematoma de volume maior que $30 \mathrm{~cm}^{3}$, a craniotomia com esvaziamento do hematoma diminui a mortalidade, apesar de não melhorar o prognóstico funcional significativamente. Portanto, em pacientes com hematomas pequenos e com nível de consciência preservado, o manejo clínico em UTI parece ser o mais coerente. Nos pacientes com hematomas com diâmetro maior que $5 \mathrm{~cm}$ ou volume maior que $30 \mathrm{~cm}^{3}$ e com comprometimento do nível de consciência, o tratamento cirúrgico pode ser benéfico (Quadro 2). De acordo com os resultados do STICH, pacientes comatosos (ECGlasgow menor que 9) com hematomas em gânglios da base ou tálamo raramente se beneficiam do tratamento cirúrgico. ${ }^{25}$

Quadro 2

Tendências para o tratamento cirúrgico do AVE hemorrágico

- Idade $<60$ anos

- Glasgow $\leq 13$ e $>5$

- Diâmetro $>5 \mathrm{~cm}$ ou volume $>30 \mathrm{~cm}^{3}$ (hematomas supratentoriais)

- Diâmetro $>3 \mathrm{~cm}$ ou volume $>16 \mathrm{~cm}^{3}$ (hematomas infratentoriais)

- Hematomas lobares (principalmente temporais) e cerebelares

- Hemisfério direito

\section{Hemorragia talâmica}

Por causa da profundidade da lesão e da grande quantidade de tecido cerebral a ser dissecado, geralmente é uma lesão não cirúrgica. Auer e cols. ${ }^{3}$ não encontraram benefício com a cirurgia. Nos casos de grandes hematomas, o prognóstico é muito reservado, a despeito da terapia adotada. Pacientes com hematoma nessa localização podem evoluir com hidrocefalia devida à compressão do terceiro ventrículo ou por hemorragia ventricular. O estado de coma pode ser em virtude da hidrocefalia, e não da hemorragia talâmica per se; portanto, a introdução de uma derivação ventricular externa (DVE) é essencial nesses casos.

\section{Hemorragia pontina}

Da Pian e cols. ${ }^{10}$ estudaram hemorragia no tronco cerebral e não encontraram diferenças entre o tratamento clínico e o cirúrgico. Apesar de hematomas maiores que $1,8 \mathrm{~cm}$ serem fatais ou causarem graves sequelas, hematomas pequenos estão relacionados com um bom prognóstico funcional em $90 \%$ dos pacientes.

\section{Hemorragia cerebelar}

Os pacientes com esse tipo de hemorragia podem apresentar-se à admissão com queixa apenas de cefaleia rapidamente progressiva, ao contrário daqueles com hematomas de tronco que se encontram em coma, na maioria das vezes. A expansão desse hematoma pode comprimir o quarto ventrículo e causar hidrocefalia aguda. A piora do nível de consciência nesses pacientes deve-se à compressão do mesencéfalo ou à hidrocefalia, e o retardo na conduta cirúrgica pode ser fatal. Nos casos em que os pacientes estão torporosos ou em coma, é prudente a realização de DVE e craniotomia ou craniectomia suboccipital de urgência ${ }^{16}$. Mesmo em pacientes com escore na ECGlasgow de 15, se o volume do hematoma ultrapassar $15 \mathrm{~cm}^{3}$ ou se seu maior diâmetro for maior que $3 \mathrm{~cm}$, pode-se realizar craniotomia/craniectomia suboccipital sem ou com ventriculostomia, dependendo se há ou não hidrocefalia na tomografia computadorizada (TC) de crânio pré-operatória. ${ }^{23}$ Nos casos de hemorragia estritamente vermiana, sugere-se tratamento conservador em pacientes com escore maior que 13 na ECGlasgow e apenas a realização de ventriculostomia em pacientes com nível de consciência mais rebaixados. Foram encontrados em um estudo prospectivo $100 \%$ de boa evolução neurológica em três meses utilizando esse protocolo. ${ }^{2}$

E se o paciente já se apresentar na admissão em coma profundo? Da Pian e cols. ${ }^{10}$ encontraram mortalidade de $100 \%$ nesses casos, mesmo nos pacientes submetidos à cirurgia de urgência, resultado semelhante encontrado por diversos autores. ${ }^{2,4,26}$ É importante salientar que alguns autores ${ }^{18}$ relatam que a perda de reflexos do tronco cerebral por compressão direta pode ser reversível. Infelizmente, ainda se necessitam de estudos controlados baseados no tempo de coma, padrão pupilar e tipo de terapêutica para saber quais os pacientes poderão se beneficiar do procedimento cirúrgico nesses casos. 


\section{Hemorragia lobar}

O tratamento específico depende do estado clínico do paciente, das possibilidades diagnósticas e da necessidade de diagnóstico histológico. Diante das inúmeras possibilidades diagnósticas nos casos de hemorragias lobares (Figuras 1 e 2), sempre que possível deve-se realizar a ressonância magnética (RM) do encéfalo ou um estudo radiológico vascular.

Em nosso Serviço, quando há dúvida no diagnóstico, é realizada uma angiotomografia, por ser um procedimento de rápida execução e menos invasivo. Nos casos de hemorragias temporais grandes, o tratamento cirúrgico de urgência torna-se imperativo, visto a possibilidade real de herniação uncal.
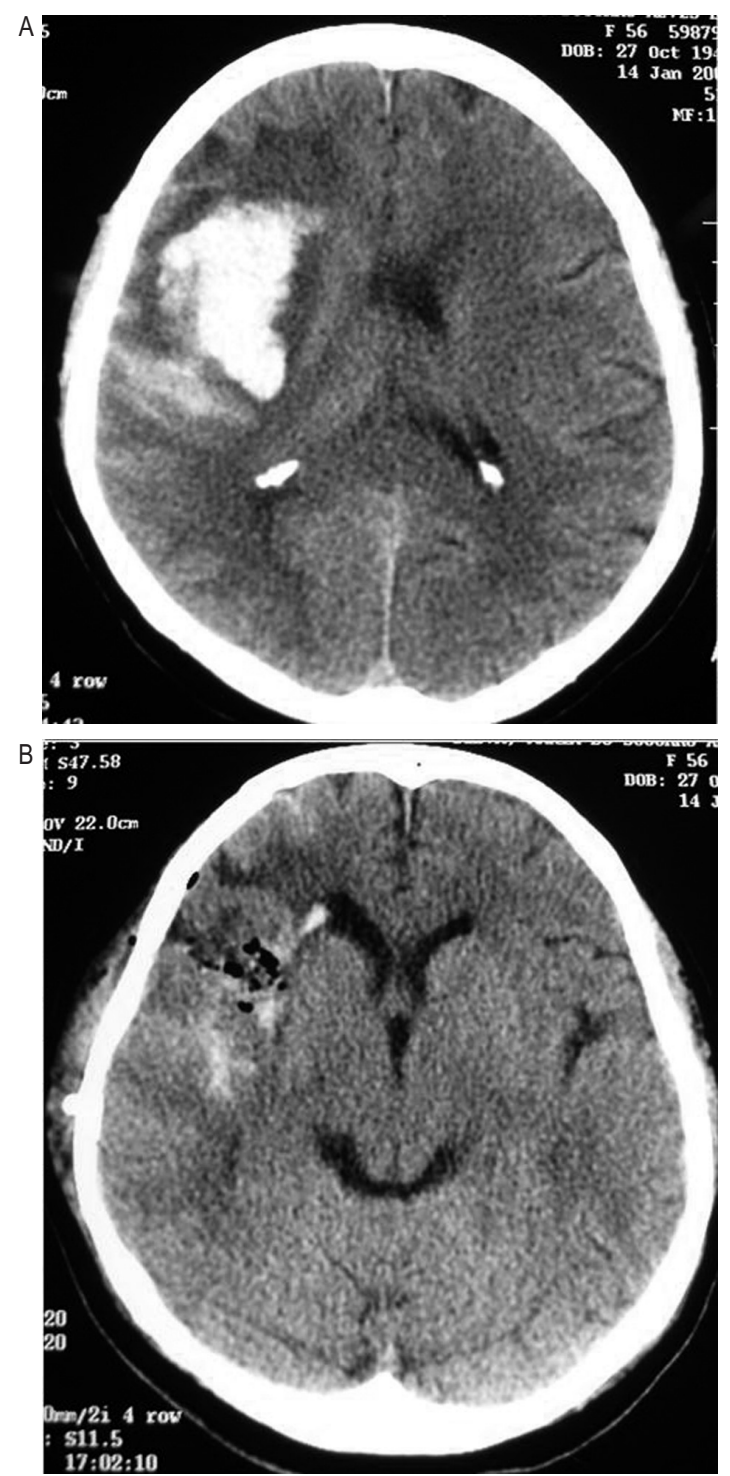

Figura 1 - (A) Hematoma lobar em paciente do sexo feminino, 56 anos, hipertensa, ECGlasgow 14, diâmetro de 4,7 cm. Optouse por tratamento cirúrgico. (B) Controle pós-operatório.

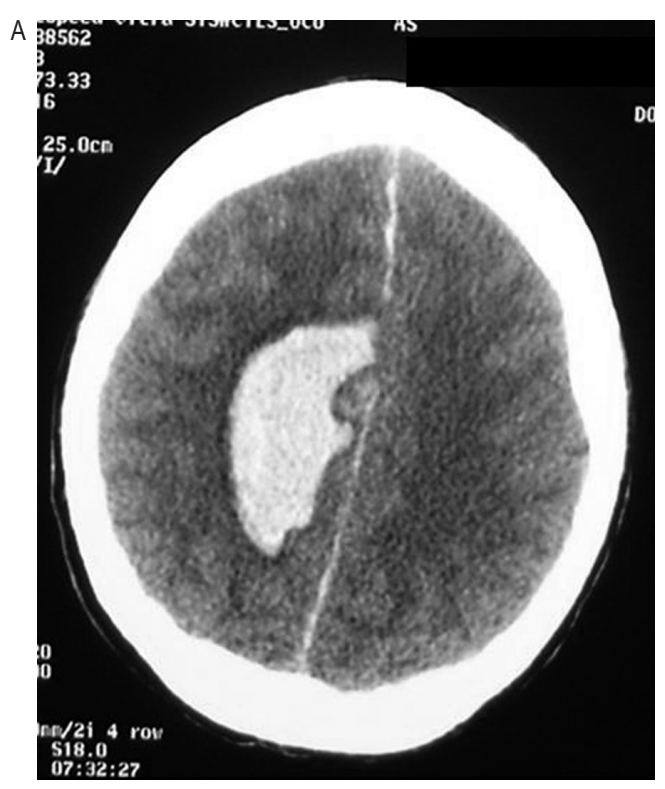

B
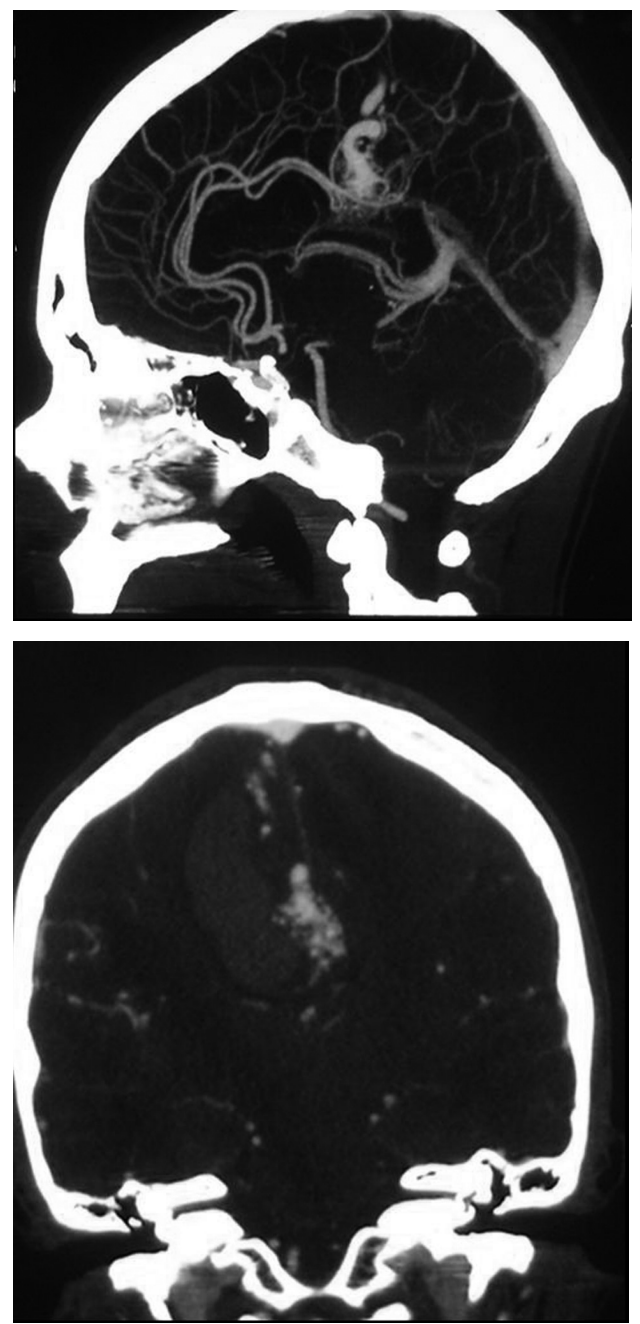

Figura 2 - (A) Hematoma frontoparietal direito em um jovem de 27 anos neurologicamente estável. ( $B$ e C) Angiotomografia evidenciando MAV medial ao giro do cíngulo. Optou-se por angiografia e programação cirúrgica em um segundo tempo. 
Na suspeita de malformação arteriovenosa (MAV), o procedimento cirúrgico somente é indicado se o paciente estiver em risco iminente de vida. $\mathrm{O}$ manejo clínico desses pacientes na fase aguda e a programação cirúrgica para ressecção da MAV parecem ser mais apropriados. Se necessária, a conduta cirúrgica na fase aguda tem como objetivo a descompressão por meio da retirada do hematoma, sem ressecção da MAV, visto que a possibilidade de ressangramento da MAV é consideravelmente menor se comparada com a dos aneurismas (4\% a 6\% em seis meses). ${ }^{15,29,33}$ Recomenda-se realizar a ressecção da MAV duas a quatro semanas após o ictus.

Se houver hemorragia em cisternas ou na fissura silviana e o paciente estiver estável neurologicamente, realiza-se angiografia para identificação de provável aneurisma cerebral. Nos casos mais graves que demandam cirurgia de emergência, pode-se realizar angiotomografia de crânio. Pasqualin e cols. ${ }^{30}$ avaliaram cerca de 300 casos de hemorragia intracerebral por ruptura de aneurisma, e o território mais acometido foi em topografia de artéria cerebral média (55\%). Eles evidenciaram que o retardo na cirurgia estava relacionado com mortalidade de $79 \%$. A cirurgia precoce determinou uma queda da mortalidade para $44 \%$. Wheelock e cols. ${ }^{40}$ evidenciaram que todos os pacientes não operados morreram e que a mortalidade caía para 29\% se o aneurisma fosse clipado na mesma cirurgia. Em um estudo prospectivo e randomizado, Heiskanen e cols. ${ }^{17}$ encontraram mortalidade de $27 \%$ nos pacientes operados em até 48 horas. Recomenda-se a realização de uma craniotomia pterional estendida. Após abertura da dura-máter, o hematoma é parcialmente removido distalmente ao aneurisma para promover o relaxamento do tecido cerebral. A drenagem de liquor da cisterna carotídea ou a lobectomia temporal pode ser alternativa para esse fim se não houver melhora. Procede-se, então, a uma dissecção transilviana com técnica microcirúrgica clássica, clipagem do aneurisma e esvaziamento do restante do hematoma. Se o cérebro estiver inchado, realiza-se a duroplastia com fáscia ou músculo. Se o inchaço persistir, sugere-se não recolocar o retalho ósseo. Há casos em que não se encontra o aneurisma. Nessa situação, a recomendação é drenar o hematoma e não recolocar o retalho ósseo.

\section{Outras modalidades de tratamento cirúrgico}

\section{Tratamento endoscópico}

Há um estudo randomizado com a avaliação de 100 pacientes em que foi comparada a aspiração endoscópica com o tratamento clínico. ${ }^{3}$ A mortalidade em seis meses no grupo cirúrgico foi de $42 \%$ e no grupo clínico foi de $70 \%$, resultado significativo estatisticamente. No caso de hematomas com volume maior do que $50 \mathrm{ml}$ especificamente, houve melhora da qualidade de vida no primeiro grupo, mas a mortalidade foi semelhante nos dois grupos. Os autores sugerem que a indicação mais precisa desse procedimento seria nos casos de hematomas lobares em pacientes com menos de 60 anos (Figura 3). Esse benefício talvez seja devido à
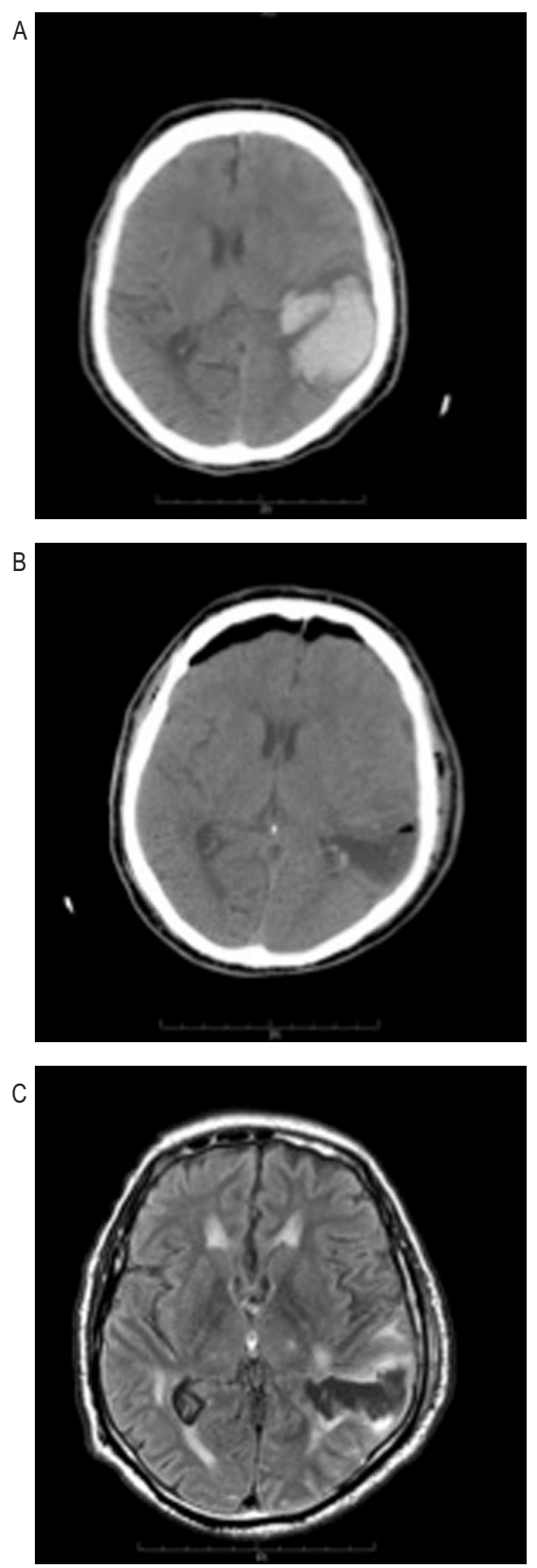

Figura 3-Paciente com TC de crânio revelando hematoma temporoparietal esquerdo (A). Realizada trepanação, seguida pela introdução de endoscópio rígido e drenagem do hematoma.

A TC pós-operatória revela bom resultado cirúrgico (B). $\mathrm{Na}$

ressonância nuclear magnética pós-operatória, sequência

FLAIR mostra o hipersinal discreto em torno da lesão denotando mínima manipulação tecidual $(C)$. 
redução do estresse cirúrgico. Entretanto, mais estudos controlados são necessários para definir o real benefício desse procedimento. Nos pacientes com hemorragia ventricular maciça e hidrocefalia, o tratamento por endoscopia pode ser uma ótima opção, pois permite a drenagem do hematoma, a realização de septostomia e a passagem de DVE sob visualização direta, diminuindo, dessa forma, a possibilidade de obstrução do sistema de derivação ventricular.

\section{Lise do hematoma com aspiração estereotáxica}

Em um ensaio multicêntrico ${ }^{37}$ em que foram avaliados 71 pacientes com hematomas supratentoriais, em que 36 receberam intervenção cirúrgica e 35 foram tratados clinicamente, não houve diferença estatística na mortalidade após seis meses $(56 \%$ e $59 \%$, respectivamente). $O$ benefício desse procedimento talvez tenha sido mascarado pela taxa de ressangramento de $22 \%$, provavelmente devido ao uso da uroquinase para lise do hematoma.

\section{Conclusões}

Enquanto a mortalidade da hemorragia intracerebral espontânea é mais alta do que os outros tipos de AVE, a abordagem imediata pode salvar o paciente. Devem-se considerar vários fatores ao se decidir pelo tratamento cirúrgico ou clínico. O nível de consciência no pré-operatório é o fator preditivo mais importante quanto à morbidade no pós-operatório. $\mathrm{O}$ resultado do procedimento cirúrgico não altera os resultados quando se comparam os pacientes em estado de alerta com os comatosos. Entretanto, mais da metade dos pacientes tem nível de consciência alterado, sendo, portanto, candidatos à cirurgia. $\mathrm{O}$ cirurgião deve instituir o tratamento antes da piora neurológica.

\section{Referências}

1. Altumbabic M, Peeling J, Del Bigio M. Intracerebral hemorrhage in the rat: effects of hematoma aspiration. Stroke. 1998;29:1917-23.

2. Auer LM, Auer T, Sayama I. Indications for surgical treatment of cerebellar haemorrhage and infarction. Acta Neurochir (Wien). 1986;79:74.

3. Auer LM, Deinsberger, Niederkorn K, Gell G, Kleinert R, Schneider G, et al. Endoscopic surgery versus medical treatment for spontaneous intracerebral hematoma: a randomized study. J Neurosurg. 1989;70:530-4.
4. Brennan R, Bergland R. Acute cerebellar hemorrhage: analysis of clinical findings and outcome in 12 cases. Neurology. 1977;27:527-30.

5. Broderick JP. Intracerebral hemorrhage. In: Gorelick PB, Alter M, editors. Handbook of neuroepidemiology. New York: Marcel Dekker; 1994, p. 141-67.

6. Broderick JP, Brott TG, Duldner JE, Tomsick T, Huster G. Volume of intracerebral hemorrhage. A powerful and easyto-use predictor of 30-day mortality. Stroke. 1993;24:987-93.

7. Broderick JP, Brott T, Tomsick T, Miller R, Huster G. Intracerebral hemorrhage more than twice as common as subarachnoid hemorrhage. J Neurosurg. 1993;78:188-91.

8. Cheung RT, Zou LY. Use of original, modified, or new intracerebral hemorrhage score to predict mortality and morbidity after intracerebral hemorrhage. Stroke. 2003;34:1717-22

9. Counsell C, Boonyakarnkul S, Dennis M. Primary intracerebral haemorrhage in the Oxforshire community project. 2. Prognosis. Cerebrovasc Dis. 1995;5:26-34.

10. Da Pian, R, Bazzan, A, Pasqualin A. Surgical versus medical treatment of spontaneous posterior fossa hematomas: a cooperative study on 205 cases. Neurol Res. 1984;6:145-51.

11. Diringer MN, Edwards DF, Zazulia AR. Hydrocephalus: a previously unrecognized predictor of poor outcome from supratentorial intracerebral hemorrhage. Stroke. 1998;29:1352-7.

12. Flemming KD, Wijdicks EF, Li H. Can we predict poor outcome at presentation in patients with lobar hemorrhage? Cerebrovasc Dis. 2001;11:183-9.

13. Fogelholm R, Avikainen S, Murros K. Prognostic value and determinants of first-day mean arterial pressure in spontaneous supratentorial intracerebral hemorrhage. Stroke. 1997;28:1396-400.

14. Gebel JM Jr, Jauch EC, Brott TG, Khoury J, Sauerbeck L, Salisbury $\mathrm{S}$, et al. Relative edema volume is a predictor of outcome in patients with hyperacute spontaneous intracerebral hemorrhage. Stroke. 2002;33:2636-41.

15. Graf CJ, Perret GE, Torner JC. Bleeding from cerebral malformations as part of their natural history. $\mathrm{J}$ Neurosurg. 1983;58:331-7.

16. Heiskanen O. Treatment of spontaneous intracerebral and intracerebellar hemorrhages. Stroke. 1993;24(12 Suppl):194-5.

17. Heiskanen O, Poranen A, Kuurne T, Valtonen S, Kaste M. Acute surgery for intracerebral haematomas caused by rupture of an intracranial arterial aneurysm. A prospective randomized study. Acta Neurochir (Wien). 1988;90:81-3.

18. Heros RC. Surgical treatment of cerebellar infarction. Stroke. 1992;23:937-8.

19. Juvela S, Heiskanen O, Poranen A, Valtonen S, Kuurne T, Kaste M, et al. The treatment of spontaneous intracerebral hemorrhage. A prospective randomized trial of surgical and conservative treatment. J Neurosurg. 1989;70:755-8.

20. Kanaya $\mathrm{H}$, Kuroda $\mathrm{K}$. Development in neurosurgical approaches to hypertensive intracerebral hemorrhage in Japan. In: Kaufman HH, editor. Intracerebral hematomas. New York: Raven Press; 1992. p. 197-210.

21. Kaneko M, Tanaka K, Shimada T, Sato K, Uemura K. Longterm evaluation of ultra-early operation for hypertensive intracerebral hemorrhage in 100 cases. J Neurosurg. 1983;58:838-42.

22. Karnik R, Valentin A, Ammerer HP, Hochfelner A, Donath P, Slany J. Outcome in patients with intracerebral hemorrhage: predictors of survival. Wien Klin Wochenschr. 2000;112:169-73.

23. Kobayashi S, Sato A, Kageyama Y, Nakamura H, Watanabe Y, Yamaura A. Treatment of hypertensive 
cerebellar hemorrhage - surgical or conservative management? Neurosurgery. 1994;34:246-51.

24. Lee JI, Nam do H, Kim JS, Hong SC, Shin HJ, Park K, et al. Stereotactic aspiration of intracerebral haematoma: significance of surgical timing and haematoma volume reduction. J Clin Neurosci. 2003;10:439-43.

25. Mendelow AD, Gregson BA, Fernandes HM, Murray GD, Teasdale GM, Hope DT, et al. For the STICH investigators. Early versus initial conservative treatment in patients with spontaneous supratentorial intracerebral haematomas in the International Surgical Trial in Intracerebral Haemorrhage (STICH): a randomized trial. Lancet. 2005;365:387-97

26. Mohadjer M, Eggert R, May J, Mayfrank L. CT-guided stereotactic fibrinolysis of spontaneous and hypertensive cerebellar hemorrhage: Long-term results. J Neurosurg. 1990;73:217-24.

27. Morgenstern LB, Demchuk AM, Kim DH, Frankowski RF, Grotta JC. Rebleeding leads to poor outcome in ultraearly craniotomy for intracerebral hemorrhage. Neurology. 2001;56:1294-9.

28. Morgenstern LB, Frankowski RF, Shedden P, Pasteur W, Grotta JC. Surgical treatment of intracerebral hemorrhage (STICH): a single-center, randomized clinical trial. Neurology. 1998;51:1359-63.

29. Ondra SL, Troupp H, George ED, Schwab K. The natural history of symptomatic arteriovenous malformations of the brain: a 24-year follow-up assessment. J Neurosurg, 1990;73:387-92.

30. Pasqualin A, Bazzan A, Cavazzani P, Scienza R, Licata C, Da Pian R. Intracranial hematomas following aneurysmal rupture: experience with 309 cases. Surg Neurol. 1986;25: 6-17.

31. Petrovitch H, Vogt TM, Berge KG. Isolated systolic hypertension: lowering the risk of stroke in older patients. SHEP Cooperative Research Group. Geriatrics. 1992; 47:30-2.

32. Probstfield JL, Applegate WB, Borhani NO, Curb JD, Cutler JA, Davis BR, et al. The Systolic Hypertension in the Elderly Program (SHEP): an intervention trial on isolated systolic hypertension. SHEP Cooperative Research Group. Clin Exp Hypertens. 1989;11:973-89.
33. Shah MV, Heros RC. Intracerebral hemorrhage due to cerebral arteriovenous malformations. Neurosurg Clin North Am. 1992;3:567-79.

34. SHEP Cooperative Research Group. Prevention of stroke by antihypertensive drug treatment in older persons with isolated systolic hypertension. Final results of the Systolic Hypertension in the Elderly Program (SHEP). JAMA. 1991;265:3255-64.

35. Steiner I, Gomori JM, Melamed E. The prognostic value of the CT scan in conservatively treated patients with intracerebral hematoma. Stroke. 1984;15:279-82.

36. Tan SH, Ng PY, Yeo TT. Hypertensive basal ganglia hemorrhage: a prospective study comparing surgical and nonsurgical management. Surg Neurol. 2001;56:287-93.

37. Teernstra OP, Evers SM, Lodder J. Stereotactic treatment of intracerebral hematoma by means of plasminogen activator: a multicenter randomized controlled trial (SICHPA). Stroke. 2003;34:968-74.

38. Wagner KR, Xi G, Hua Y. Ultra-early clot aspiration after lysis with tissue plasminogen activator in a porcine model of intracerebral hemorrhage: edema and blood-brain barrier protection. J Neurosurg. 1999;90:491-8.

39. Wang X, Mori T, Sumii T. Hemoglobin-induced cytotoxicity in rat cerebral cortical neurons: caspase activation and oxidative stress. Stroke. 2002;33:1882-8.

40. Wheelock B, Weir B, Watts R. Timing of surgery for intracerebral hematomas due to aneurysm rupture. J Neurosurg. 1983;58:476-81.

41. Zazulia AR, Diringer MN, Derdeyn CP. Progression of mass effect after intracerebral hemorrhage. Stroke. 1999;30: 1167-73.

42. Zuccarello M, Brott $T$, Derex L. Early surgical treatment for supratentorial intracerebral hemorrhage: a randomized feasability study. Stroke. 1999;30:1833-39.

\section{Endereço para correspondência}

Robson Luis Oliveira de Amorim

Rua Alves Guimarães, 642, ap. 82

05410-001 - São Paulo, SP, Brasil

Telefones: (11) 9134-4180/(11) 3085-4446

E-mail: amorim.robson@uol.com.br 